\title{
Undercover-Religion in James Camerons Film "Titanic"
}

„Doch es wird ein Unheil über dich kommen, das du nicht wegzaubern kannst. Ein Verderben wird dich überfallen, das du nicht zu bannen vermagst. Und plötzlich wird dein Untergang kommen, an den du niemals gedacht hast." 1 Jes 47,11

\section{Religiös-mythische Dimensionen des Untergangs der "Titanic"}

„Es ist der überwölbende Sinnhorizont gesellschaftlicher Kommunikation, der Katastrophenmeldungen erst zu solchen macht", schreibt Wilhelm Hofmann. „Ein Erdbeben ist eben nicht nur ein "Naturereignis', sondern es kann unter bestimmten Voraussetzungen mehr als eine Stadt, nämlich ein ganzes Weltbild erschüttern, und eine Überschwemmung wird unter Umständen zur Strafe Gottes für eine außer Kontrolle geratene Schöpfung (Genesis 6-8)." 2 So verhielt es sich auch mit dem Untergang der „Titanic“, der auf drastische Weise verdeutlichte, dass es Kräfte auf dieser Welt gibt, die sich dem menschlichen Einfluss entziehen. Vielen erschien die Schiffskatastrophe daher als Strafe Gottes für die Hybris der Technik.

Frances Fisher, die in James Camerons „Titanic“ Ruth, die Mutter der Protagonistin Rose, verkörpert, sagt über den Untergang des Luxusschiffes: „,God is greater than all of us, and if you are so arrogant as to say something is unsinkable, you will get slapped in the face "“3, und der Presbyterianer Charles H. Parkurst bezeichnet das Sinken der "Titanic“ als "terrific and ghastly illustration of what things come to when men throw God out of the door". ${ }^{4}$ Kardinal Franz König vergleicht die westliche Welt mit den Passagieren der „Titanic“ wenn er sagt: „Die Industriestaaten der westlichen, ehemals christlichen Welt leben heute wie jene Luxusgesellschaft, die auf der ,Titanic fuhr, als sie in den Fluten ver-

2 Hofmann, Wilhelm: Nichts ist so unterhaltend wie der Untergang der Welt. Überlegungen zur Risikokommunikation in Katastrophenfilmen. In: ders. (Hg.): Sinnwelt Film. Beiträge zur interdisziplinären Filmanalyse. Baden-Baden 1996, S. 159-193, hier S. 163.

3 Fisher, Frances In: Marsh, Ed/Kirkland, Douglas/Cameron, James: James Cameron's Titanic. New York 1997, S. 165.

4 Parkhurst, Charles H. In: Biel, Steven: Titanica. The Disaster of the Century in Poetry, Song, and Prose. New York 1998, S. 41. 
sank. Die Bordkapelle unserer Erde spielt bis zum letzten Augenblick zum Tanz auf, zum Luxustanz, zum Wegwerftanz, zum Tanz der Verschwendung und der Vergeudung. Der reiche Prasser beginnt heute die Tage seines letzten Festes. Inzwischen stirbt Lazarus vor seiner Haustür, ohne daß es jemand bemerkt." 5

Auch als Gegenbild der Arche Noah (Gen 6-8) kann die "Titanic“ gesehen werden. Während die Arche aufgrund göttlicher Anweisungen als ein Rettungsboot entstand, wurde die "Titanic" aus dem selbstsicheren Geist menschlicher Schöpfungsmacht heraus gebaut. Eine weitere Verbindung zu biblischen Erzählungen findet sich beim Vergleich des Baues der „Titanic“ und des Turms zu Babel (Gen 11,1-9). Der biblische Turmbau verdeutlicht ebenso wie der Bau der "Titanic" die Hybris, die größenwahnsinnige Überheblichkeit des Menschen über seine Grenzen ${ }^{6}$, die sich im Willen manifestiert, eine unïbertreffliche Kulturleistung zu schaffen, die durch Größe und Rekorde beeindruckt. ${ }^{7}$ Noch heute fasziniert der „Mythos ,Titanic““ als Sinnbild aller Katastrophen unseres Jahrhunderts, als ein fassbares Bild für den Verlust einer Welt voller Optimismus und den Glauben an die Zukunft, den Fortschritt und die Wunder der Technik. Trotz unserer vermeintlichen Herrschaft über die Natur sind wir, so C. G. Jung, „immer noch wie eh und je ihre Opfer und haben noch nicht einmal gelernt, unsere eigene Natur in den Griff $z u$ bekommen, die langsam, aber unausweichlich der Katastrophe entgegensteuert" 8

\section{2. „Titanic"-Kult}

Das Gefühl, von einer Macht beherrscht zu werden, der wir ausgeliefert sind und die unser Schicksal gänzlich unserem Einwirken entziehen kann, führt zu einem quasi-religiösen Gefühl, das die heutigen Katastrophen-Partizipanten beschleicht, wenn sie sich etwa einen Kinofilm wie „Titanic“ zu Gemüte führen. Darin liegt auch einer der Gründe für die Faszination der „Titanic“-Artefakte und für die Reliquienverehrung, die

5 Kardinal Franz König. In: Gartlgruber, Peter / Weinhandl, Helfried: Wie leben? Schul- und Werkbuch für den Religionsunterricht. 3. Aufl. Wien 1991, S. 24. (Zum Gleichnis vom armen Lazarus siehe Lk 16,19-31.)

6 In Camerons Film wird die Hybris unterstrichen durch eine Bemerkung von Roses arrogantem Verlobten Cal: "It is unsinkable. God himself could not sink this ship."

7 Zudem führte die „Titanic“ aus ästhetischen Gründen nicht genug Rettungsboote mit sich. Weiterhin wurde die Fahrtgeschwindigkeit gesteigert, in der Hoffnung, das blaue Band zu erlangen.

8 Jung, Carl Gustav: Gesammelte Werke. Band 18/1: Das symbolische Leben. Olten 1981, S. 282. 
in den „Titanic“-Ausstellungen zum Ausdruck kommt. „Titanic“-Ausdrucksartefakte „bieten insgesamt eine seltsame Mischung aus Realismus und Katharsis, aus Erinnerungskult, Kitsch und Religiosität“. ${ }^{9}$ Hans Magnus Enzensberger ist der Ansicht, das Ganze wirke „wie ein technisch-religiöser Fetisch-Kult". ${ }^{10}$ So zeigt etwa eine Postkarte, auf der die sinkende "Titanic" abgebildet ist, eine zum Himmel blickende Frau und Gott, der vom Himmel auf das sinkende Schiff herabsieht. Die Karte trägt folgenden Schriftzug: „,Save, Lord, we perish', was their cry, , 0 save us in our agony! Thy word above the storm rose high ,Peace, be still." "11

Der Verein „Titanic Historical Society“ (T.H.S.) mit seiner Zeitschrift "The Titanic Commutator" als regelmäßig erscheinendes Organ befasst sich noch heute mit den Geschehnissen rund um das Luxusschiff. Ein Leserbrief im „Titanic Commutator" lässt die eindeutig religiösen Züge der „Titanic“-Verehrung erkennen: „Laßst uns froh sein über das, was wir von ihr gelernt haben. Sie bezahlte den Preis, damit wir davon profitieren können. Lang lebe unsere heilige Jungfrau, die Titanic!"12 So bietet der „Titanic“-Kult in gewisser Weise einen Ersatz für Religion. Der Kitsch, in den der Kult gekleidet ist, ist elementarer Bestandteil des „,Titanic“Glaubens": Kitsch, so Georg Seeßlen, ,wird um so dringlicher notwendig, als die Kirche und ihre Kultur dem einzelnen nicht mehr die Angst abnimmt". ${ }^{13}$

9 Grimm, Jürgen: Das Titanic-Phänomen. Untergangsmythos zwischen Kitsch und Katharsis. In: Gemeinschaftswerk der evangelischen Publizistik e.V: Medien praktisch. Texte. Filmerleben. Zur emotionalen Dramaturgie von Titanic. Sonderheft Nr. 2 der Zeitschrift medien praktisch, September 1998, S. 17-29, hier S. 21.

10 „Die Natur schlägt zurück!“. Erik Fosnes Hansens Protokoll eines „Titanic“Fachgesprächs mit Hans Magnus Enzensberger. In: Der Spiegel, Nr. 13/1998, S. 238-239, hier S. 239.

11 Bamforth Memorial Postcard (22). In: Bown, M./Simmons, R.: R.M.S. ,Titanic'. A portrait in old picture postcards. Seaford, East Sussex: S. B. Publications (erstmals 1987). Zit.n.: Grimm, Jürgen: Das Titanic-Phänomen. Untergangsmythos zwischen Kitsch und Katharsis. In: Gemeinschaftswerk der evangelischen Publizistik: Medien praktisch. Texte. Filmerleben. Zur emotionalen Dramaturgie von Titanic. Sonderheft Nr. 2 der Zeitschrift medien praktisch, September 1998, S. 17-29, hier S. 22, Abbildung 4.

12 Rauscher, William. In: „The Titanic Commutator“. Zit.n.: ebd., S. 22.

13 Seeßlen, Georg: Kino der Gefühle. Geschichte und Mythologie des Film-Melodrams. Reinbek bei Hamburg 1980, S. 27. 


\section{Vertiefung des "Titanic"-Kultes durch James Camerons Film "Titanic"}

Angesichts dieser Voraussetzungen liegt es nahe, dass ein Film über den Untergang der „Titanic“ ein eigenes Glaubenssystem etablieren und dass selbst die Rezeption eines solchen Filmes kultische Züge tragen kann. Tatsächlich wurde James Camerons „Titanic“ zu einem Medienereignis, das alle Anzeichen einer Religion enthält. „Es setzt sich wellenförmig in immer weitere Bereiche fort, erfasst Menschen, die noch vor kurzem entweder das Kino schon ein für allemal verlassen $z \mathfrak{u}$ haben schienen oder sich als definitiv kitsch-resistent wähnten. Im Umkreis der medialen Ersatzreligion gibt es nichts Triviales, nichts Unbedeutendes mehr." 14

Vielen erscheint die „Titanic“ als Symbol für die gegenwärtige Lage der Welt. Während eine 30-Jährige nach der Kinovorstellung sinniert: „Das Schiff ist wie die Welt heute, sie steuert auch dem Untergang $z u$, alle wissen es, und niemand kann etwas dagegen tun"15, erkennt Georg Seeßlen in Camerons Film ,eine Rettungsphantasie für eine Gesellschaft, in der man sich nicht einmal mehr gegenseitig helfen mag". ${ }^{16}$ Weiter kontrastiert "Titanic" Liebesgeschichte und Katastrophengeschehen, wobei beide Motivkomplexe vielfach religiös aufgeladen sind. Stephan Vasel sieht in der Tatsache, daß der erfolgreichste Film aller Zeiten von religiösen Motiven durchzogen ist, einen „äußerst wichtigen Indikator für die religiöse Lage der Gegenwart". ${ }^{17}$ Auf einen weiteren Indikator für die religiöse Situation der westlichen Welt stößt man beim Blick in die Zeitschrift „Premiere“, die, als abzusehen war, dass „Titanic“ die Milliarden-Dollar-Grenze an den Kinokassen sprengen würde, einen Artikel mit dem Titel "Cameron is God" veröffentlichte. ${ }^{18}$

14 Ders.: Der Höhenflug des sinkenden Schiffs: Das Medienereignis „Titanic“. In: epd Film. 15. Jg. 5/1998, S. 8-9, hier S. 9.

15 Woltron, Ute: Delphine im Eismeer. In: Profil Nr. 14 / 30.3.1998, S. 108-109, hier S. 109. James Cameron wollte diese Parallele ursprünglich durch eine Drehbuchzeile hervorheben: „In a line af dialogue that Cameron cut from the script before shooting began, Old Rose admonished Brock, ,There's another iceberg out there, Mr. Lovett. I don't know what it is ... but I do know the force driving us toward it" (Marsh, Ed / Kirkland, Douglas / Cameron, James: James Cameron's Titanic. New York 1997, S. 172).

16 Seeßlen, Georg: Der Höhenflug des sinkenden Schiffs: Das Medienereignis „Titanic“. In: epd Film. 15. Jg. 5/1998, S. 8-9, hier S. 9.

17 Vasel, Stephan: Kultkino und Religion. Christliches Erbe im Film am Beispiel „Titanic“. In: Materialdienst der Evangelischen Zentralstelle für Weltanschauungsfragen. 63. Jg. 12/2000, S. 411-428, hier S. 426.

18 Thompson, Anne: Cameron is God. In: Premiere 4/1998, S. 63f. und 110. 


\section{Die Liebe als Element der Glaubensbotschaft „Titanic's"}

Gelingende Liebe stellt „das zentrale Credo der Religion des Kinos der Jahrtausendwende" dar. ${ }^{19}$ Auch dem Film "Titanic" liegt ein Glaubenssatz über die Liebe zugrunde: „Das Leben macht Sinn, sofern einem ein einziges Mal die wahre Liebe begegnet ist." 20 Stephan Vasel bemerkt, theologisch gesprochen sei „Titanic“ „ein Film über die Sünde, der die Liebe als einzig adäquates Verhaltensmuster in der gefallenen (hier sinkenden) Welt beschreibt". ${ }^{21}$ James Cameron erkannte den Zusammenhang zwischen „Titanic's“ Botschaft der Liebe und der menschlichen Sehnsucht nach einer höheren, lenkenden Kraft, als er sagte: „Wenn man jemanden liebt, kann man sich ein Ende dieser Liebe nicht vorstellen ..., daß man also nicht irgendwann wieder vereinigt wird. Ich halte das für ein elementares psychologisches Bedürfnis, das die Wurzel von Spiritualität ist." 22

Diesem - heute aufgrund vagierender Religiosität vielfach heimatlosen - Bedürfnis kommt Cameron entgegen, indem er mit der Botschaft „Titanic's" eine Glaubensaussage trifft, die ihre Wirkung unabhängig von jeglicher Religionszugehörigkeit entfaltet: Der Glaube an den Beistand Gottes „wird abgelöst durch die Unbedingtheit und Maßlosigkeit einer Liebe, kraft derer Menschen sogar die Schranke des Todes - psychologisch - zu überwinden vermögen. Vertrauen in den geliebten Menschen überwölbt im ... parallelen Vertrauensdiskurs als Säkularisierung das Gottvertrauen“23 (vgl. dazu auch: 1 Joh 4,7-8). „Titanic“, so Jörg Hermann, „verkündigt das Evangelium einer Liebe, die stärker ist als der Tod." 24 Da-

19 Herrmann, Jörg: Die Liebe ist stark wie der Tod. Religiöse Motive im zeitgenössischen Film. In: Zeitzeichen. Evangelische Kommentare zu Religion und Gesellschaft. 2. Jg. 5/2001, S. 48-50, hier S. 50.

20 Vasel, Stephan: Kultkino und Religion. Christliches Erbe im Film am Beispiel "Titanic“. In: Materialdienst der Evangelischen Zentralstelle für Weltanschauungsfragen. 63. Jg. 12/2000, S. 411-428, hier S. 423.

21 Ebd., S. 424.

22 Titanic Story Book: James Camerons illustriertes Drehbuch; ausführliches Interview mit Autor, Regisseur und Produzent James Cameron, kommentiert von Randall Frakes. Nürnberg 1999, S. 153.

23 Behr, Manfred: Philologisches Filmerleben. Literarische Dimensionen in Titanic. In: Gemeinschaftswerk der evangelischen Publizistik: Medien praktisch. Texte. Filmerleben. Zur emotionalen Dramaturgie von Titanic. Sonderheft Nr. 2 der Zeitschrift medien praktiach, September 1998, S. 52-64, hier S. 63.

24 Herrmann, Jörg: Die Liebe ist stark wie der Tod. Religiöse Motive im zeitgenössischen Film. In: Zeitzeichen. Evangelische Kommentare $\mathrm{zu}$ Religion und Gesellschaft. 2. Jg. 5/2001, S. 48-50, hier S. 49. (Rose, die ihr Überleben Jack 
durch steigert Cameron selbst den biblischen Text des Hohenlieds, in dem es heißt: „Stark wie der Tod ist die Liebe“ (Hld 8,6) und erhebt die Liebe in den Status einer religionsäquivalenten Sinnvermittlungsinstanz, denn es sind allein die Liebenden, die füreinander das leisten, was im Christentum von Gott ausgeht.

\section{Das Opfer als Element der Glaubensbotschaft „Titanic's"}

Die Botschaft der befreiend-erlösenden Kraft der Liebe reicht in "Titanic“ bis zu jener von Selbstlosigkeit und Hingabe geprägten Höchstform der Liebe, die den eigenen Vorteil zugunsten des jeweils anderen vergisst. So gewinnt das Opfer als Lebenshingabe Bedeutung im Kontext der Beziehung der Liebenden. Indem Jack und Rose sich unter Einsatz der eigenen Existenz mit der Gefahr des Selbstverlusts aufeinander einlassen, trifft ihre Liebe strukturell eine, wenn nicht sogar die Grundbestimmung des Christlichen.

Der häufige Standardmythos aktueller Kinofilme von der Rettung oder Befreiung der Welt vom Bösen durch einen sagenhaften Erlöser, der sich häufig selbst dabei opfert, findet sich in gebrochener Form bei James Camerons „Titanic“, der, so Ritter, „auf seine Weise den Erlösungs- und Auferstehungsmythos neu inszeniert". ${ }^{25}$ Indem ein Opfer, die Lebenshingabe des geliebten Menschen sowie dessen „Auferstehung“ als Inrechtsetzung des Opfers gezeigt werden und all das zudem die ebenfalls in „Titanic“ präsenten Systeme von Macht, Geld und Größenwahn kontrastiert, zitiert und montiert Cameron zentrale Stationen der Heilsgeschichte des Christentums.

\section{Christologisch-soteriologische Attribute des Jack Dawson als Element der Glaubensbotschaft „Titanic's"}

„Titanic" erzählt von einer heroischen Opfertat zugunsten eines anderen Menschen. In der Filmhandlung finden sich daher Parallelen zur Selbst-

verdankt, der sein Leben für sie hingab, bleibt ihrem Geliebten auch nach dessen Tod treu. Sie nimmt seinen Namen, Dawson, an und erfüllt die Versprechen, die sie dem sterbenden Jack einst gab, indem sie überlebt, Kinder bekommt und als alte Frau nach einem erfüllten Leben friedlich in ihrem Bett stirbt.)

25 Ritter, Werner H.: Mythos mit Thrill. Das Kino nimmt sich religiöser Erlösungs Motive an. In: Zeitzeichen. Evangelische Kommentare zu Religion und Gesellschaft. 2. Jg. 1/2001, S. 53-55, hier S. 53. Siehe dazu auch: ders.: Abschied vom Erlöser-Mythos? In: Zeitschrift für Pädagogik und Theologie Nr. 1, Jg. 35/2001, S. 68-75, hier S. 69. 
opferung Jesu zugunsten der Menschheit. So liegt es nahe, die Figur des Jack Dawson als Helden zu sehen, der deutliche Christus-Züge aufweist. Rettete Christus die Menschheit durch seine Liebe und Selbstopferung, so rettete Jack Rose ebenso durch seine Liebe und die Hingabe seines Lebens. Dabei hat das Credo in Camerons Film den Vorteil, dass es, während heute angesichts der gesellschaftlichen, politischen, umweltspezifischen und gentechnischen Lage nur mehr ein kleiner Teil der Menschheit die Errettung durch Jesus glauben kann, für viele leichter anzunehmen ist, dass Jack Rose errettet. Die Hoffnung auf Erlösung wird erfüllt, zwar im kleinen Rahmen, eben deshalb aber auch in für viele Menschen und unabhängig von deren Religionszugehörigkeit glaubhafter Form.

Die Szene, in der Jack und Rose mit ausgebreiteten Armen in Form eines Doppelkreuzes am Bug der „Titanic“ stehen, verdichtet die Verweise von der Figur des Jack Dawson auf Jesus Christus. ${ }^{26}$ Wie Jesus ist auch Jack ein materiell armer, aber fröhlicher Mann, ein Vagabund mit nur gelegentlichem festen Wohnsitz. Jacks Reise auf der "Titanic“ wird sich als sein persönlicher Kreuzesweg erweisen. Genau wie einst Jesus wird Jack für seine Hilfe nicht gedankt, als er Rose vor dem Suizid errettet, wie Jesus wird Jack, der ebenfalls aus Liebe handelt, zu unrecht angeklagt, in Ketten gelegt und verspottet. Mehrfach gibt es Tötungsbeschlüsse gegen ihn (sowohl Cals Diener Lovejoy als auch Roses Verlobter Cal erschießen ihn beinahe), während er selbst mehrfach als (Lebens-)Retter auftritt (Rose ${ }^{27}$, kleines Kind).

Während des vornehmen Dinners der Ersten Klasse lehnt Jack den ihm angebotenen Kaviar ab und beißt stattdessen in ein brotartiges Gebäck. Danach erhebt er sein Glas zu einem Trinkspruch. Diese Gesten lassen Parallelen zum letzten Abendmahl Jesu und seiner Jünger anklingen (vgl. Mt 26,26-27, Mk 14,22-23, Lk 22,19-20). Brot steht symbolisch für die lebenserhaltenden Kräfte bzw. für das Leben selbst. ${ }^{28}$ Neben der Betonung des Verwurzeltseins Jacks mit dem Leben und des dadurch stark hervorgehobenen Kontrasts zur lebensfremden Etikette der Ersten Klasse, ergeben sich auch Parallelen zur Selbsthingabe Jesu, der sich

26 Dazu Adele Reinhartz: „One does not need a degree in biblical studies to understand that a person...standing cross-like with out-stretched arms, might be a savior-figure" (Reinhartz, Adele: Scripture on the Silver Screen. In: The Journal of Religion and Film, Vol. 3, No. 1/1999. In: http://www.unomaha.edu/ ${ }^{\sim}$ wwwjrf/scripture.htm am 10.4.2002).

27 So sagt die 10 -jährige Rose über Jack: „He saved me - in every way that a person can be saved."

28 Vgl: Lurker, Manfred: Die Botschaft der Symbole. In Mythen, Kulturen und Religionen. München 1990, S. 272. 
auch „Brot des Lebens nannte“, symbolisiert durch das Brechen und Teilen des Brotes ${ }^{29}$ - und der Selbsthingabe Jacks aus Liebe zu Rose.

Wie Jesus ist auch Roses Retter Jack nicht von ihrer bisherigen Welt (vgl. Joh 18,36a: „Jesus antwortete: mein Königtum ist nicht von dieser Welt“). Auf Pilatus' Frage, ob Jesus ein König sei, antwortet dieser: „Du sagst es, ich bin ein König" (Joh 18,37a). Jack läßt uns dies ebenfalls wissen, als er am Bug stehend „I'm the king of the world!" ruft. ${ }^{30}$ Zudem lebt Jacks Geist weiter in Rose und trägt somit ebenso wie der Geist Christi Züge der Unsterblichkeit. Jacks Geschick und Wirken können daher christologisch-soteriologische Attribute beigemessen werden. ${ }^{31}$

Vom durch den Kapitän geleiteten Gottesdienst ${ }^{32}$ wird der moderne Christus Jack bezeichnenderweise ausgeschlossen, und als Jack und Rose zum Bug des sinkenden Schiffes eilen, versperrt ihnen ein langsam gehender, Psalm 23 betender Mann den Weg. Der Mann betet: „Yea, though I walk through the valley of the shadow of death, I will feel no ...", worauf Jack inm zuruft: „You wanna walk a little faster throught that valley there?" Diese beiden Szenen können als Anspielung auf die Dekonstruktion althergebrachter, vielleicht, so der Unterton der Szenen, überholter Glaubenssysteme zugunsten der Errichtung eines neuen Glaubenssystems gesehen werden, in dem andere Prioritäten gesetzt werden und alte Verbindlichkeiten neuen weichen. Zudem wird deutlich, dass die Kirche im Glaubenssystem „Titanic's“ keineswegs die Heimat des modernen Christus Jack ist. ${ }^{33}$ Cameron greift dadurch geschickt den Trend zur vagabundierenden Religiosität auf, wodurch er viele Menschen anspricht.

29 Zur Brotsymbolik im Christentum siehe: Forstner, Dorothea / Becker, Renate (Hg.): Neues Lexikon christlicher Symbole. Innsbruck, Wien 1991, S. 80-88.

30 James Cameron selbst verwendete angesichts der elf Academy Awards, mit denen "Titanic" ausgezeichnet wurde, Jacks Ausruf in seiner Dankesrede und krönte sich damit selbst zum „König der Welt“.

31 Vgl. dazu auch: Vasel, Stephan: Kultkino und Religion. Christliches Erbe im Film am Beispiel „Titanic“. In: Materialdienst der Evangelischen Zentralstelle für Weltanschauungsfragen. 63. Jg. 12/2000, S. 411-428, hier S. 420.

32 Die bei der Kapitänsmesse von den Passagieren der ersten Klasse gesungene Hymne: "Almighty Father Strong to Save, Whose Arm Doth Blind the Restless Wave" mit dem Refrain: "O hear us when we cry to thee for those in peril on the sea" ist ein altes englisches Kirchen- und Seemannslied. (Siehe dazu auch: Behr, Manfred: Philologisches Filmerleben. Literarische Dimensionen in Titanic. In: Gemeinschaftswerk der evangelischen Publizistik: Medien praktisch. Texte. Filmerleben. Zur emotionalen Dramaturgie von Titanic. Sonderheft Nr. 2 der Zeitschrift medien praktisch, September 1998, S. 52-64, hier S. 62.)

33 Siehe dazu auch: Vasel, Stephan: Kultkino und Religion. Christliches Erbe im Film am Beispiel „Titanic“. In: Materialdienst der Evangelischen Zentralstelle für Weltanschauungsfragen. 63. Jg. 12/2000, S. 411-428, hier S. 426. 
Da Jack „der Messias eines neuen Lebens“34 ist, muß er folgerichtig schließlich sein eigenes Leben hingeben. So wird ein weiterer Verweis von Jack auf Jesus deutlich, wenn man sich das Sterbeverhalten dieser beiden Personen verdeutlicht: Obwohl es Jack zuletzt im eiskalten Ozean schlechter geht als Rose, ist er es, der Trost spendet. Noch im Angesicht des Todes bekräftigt Jack sein die Geliebte rettendes Selbstopfer und stellt die Bedeutung der Liebe über das eigene Leben, als er sagt, das Schiffsticket $\mathrm{zu}$ gewinnen, sei das Beste gewesen, was ihm je passiert sei, da es ihn zu Rose gebracht habe. Jack stirbt mit friedlichem Gesichtsausdruck, oder, wie Stephan Vasel es formuliert, er "stirbt vorbildlich, geradezu ,selig'“. 35

Zwar bleibt Rose das gemeinsame Leben mit Jack verwehrt; dennoch ist sein Tod nicht sinnlos und bedeutet nicht das Ende ihrer Beziehung. Folgende Passage des 1. Johannesbriefes trifft zugleich eine zentrale Aussage „Titanic's“: „Wer nicht liebt, hat Gott nicht erkannt; denn Gott ist die Liebe. Die Liebe Gottes wurde uns dadurch offenbart, daß Gott seinen einzigen Sohn in die Welt gesandt hat, damit wir durch ihn leben. Nicht darin besteht die Liebe, daß wir Gott geliebt haben, sondern daß er uns geliebt hat und seinen Sohn als Sühne für unsere Sünden gesandt hat" (1 Joh 4,8-10). Im Kontext des Filmes „Titanic“ bedeutet dies: Indem Rose durch Jack die wahre Liebe erleben durfte, ist ihr das Höchste widerfahren, was im Glaubenssystem des Films möglich ist. Jacks Tod war wie der Tod Jesu nicht umsonst, denn Rose lebt weiter durch Jack.

\section{Die Betonung der Macht der Natur als Element der Glaubens- botschaft "Titanic's"}

In Zeiten zunehmender Ambivalenz des wissenschaftlichen Fortschritts tut es gut, dass das Glaubenssystem „Titanic's“ auch eine naturmythische Komponente enthält. Diese wird geschaffen durch Betonung der Naturmacht, die hier den Status der Gottesmacht einnimmt. Übermittelt wird dieser Aspekt des „Titanic"-Glaubens etwa durch eine aus der Highangle-Perspektive und aus großer Entfernung gefilmten Einstellung, in

34 Brinkmann, Thomas Frank: Die Liebe höret nimmer auf... oder Titanic und die Bergung des „Herzens“. In: Schaeffer-Brinkmann, Bettina: Kino statt Kirche? Zur Erforschung der sinngewährenden und religionsbildenden Kraft populärer zeitgenössisch-r Filme. Rheinbach 2000, S. 235-243, hier S. 240.

35 Vasel, Stephan: Kultkino und Religion. Christliches Erbe im Film am Beispiel „Titanic“. In: Materialdienst der Evangelischen Zentralstelle für Weltanschauungsfragen. 63 . Jg. $12 / 2000$, S. 411-428, hier S. 417. 
der man die angesichts der unendlichen Weite des Atlantiks relative Winzigkeit des Luxusschiffes sieht. Es scheint, als blickeder Himmel auf die Katastrophe herab, in der das vormals als überaus prächtig vorgeführte Schiff nun wie eine Nußschale in den unendlich wirkenden Weiten des Ozeans treibt. Die Kameraperspektive scheint hier an eine höhere Macht zu gemahnen. Weiter wird diese Naturmacht durch High-angleKameraperspektiven während des Untergangs der „Titanic“ betont sowie durch Leuchtraketen, die ungesehen und ungehört bleiben, mittels Bildern der fassungslos aus den Rettungsbooten das Sinken der "Titanic" beobachtenden Passagiere sowie durch die Szene, in der Rose nach dem Sinken des Schiffes auf einer Holztür im Wasser treibend leise das Lied „Come Josephine, In My Flying Machine“ singt, wonach im Gegenschuß der klare Sternenhimmel in den Blick kommt. Diese Bilder erinnern an Schleiermachers Beschreibung der religiösen Kernerfahrung als „Sinn und Geschmack fürs Unendliche“ oder „Anschauung des Universums“. ${ }^{36}$

\section{Undifferenziertes Präsenthalten der Gottesthematik als Element der Glaubensbotschaft "Titanic's"}

"Titanic" wird außerdem von etlichen weiteren religiösen Anspielungen durchzogen, wobei diese ,in dem Feld zwischen Abwesenheit und Anrufung Gottes oszillieren". ${ }^{37}$ Initiiert wird der Gottesdiskurs ausgerechnet von $\mathrm{Cal}$, der Personifikation des Bösen, als er mit blasphemischem Unterton bemerkt, die „Titanic“ sei „unsinkable. God himself could not sink this ship." Beim Dinner der Ersten Klasse erzählt Jack: „Well, right now my address is the RMS ,Titanic", after that I'm on God's good humour." Weitere Erwähnungen Gottes - wenn auch eher flüchtiger Natur - halten die Gottesthematik präsent: ein verärgertes „Goddammit!“, als der Lift boy sich weigern will, Rose mit dem bereits halb überflutenden Lift in das Unterdeck zu bringen und Passagierin Molly Browns Ausruf „God almighty! “ beim Anblick des Ausmaßes der „Titanic“-Katastrophe.

Eine weitere Zutat zum Glaubenskuchen „Titanic's“ liefert die Musik: Das Streichorchester auf dem sinkenden Schiff spielte etlichen

36 Schleiermacher, Friedrich D. E.: Über die Religion. Reden an die Gebildeten unter ihren Verächtern. Stuttgart 1980, S. 36, 80 und 85. Zit.n.: Herrmann, Jörg: Sinnmaschine Kino. Sinndeutung und Religion im populären Film. Gütersloh 2001, S. 203.

37 Behr, Manfred: Philologisches Filmerleben. Literarische Dimensionen in Titanic. In: Gemeinschaftswerk der evangelischen Publizistik: Medien praktisch. Texte. Filmerleben. Zur emotionalen Dramaturgie von Titanic. Sonderheft Nr. 2 der Zeitschrift medien praktisch, September 1998, S. 52-64, hier S. 62. 
übereinstimmenden Überlieferungen zufolge als letztes Stück den romantischen Choral "Nearer My God to Thee" 38 aus dem 19. Jahrhundert. Cameron greift dies auf und schafft so ein bitter-ironisches Sinnbild für das Scheitern der promethischen Leistung und für eine letzte Sehnsucht nach Gott. ${ }^{39}$ Auch die Selbstlosigkeit, mit der man den unmittelbar bevorstehenden Tod transzendieren kann, hat Cameron durch diese Szene aufgezeigt. ${ }^{40}$ So werden über die religiöse Komponente des Choralhaften die Filmbilder um eine existentielle Dimension erweitert.

Auf dem sinkenden Schiff schart Pater Byles einige Gläubige um sich, denen er Verse aus der Offenbarung des Johannes mit auf den Weg gibt. Jack und Rose eilen an ihm und den Gläubigen vorbei, um zum Heck des Schiffes zu gelangen. Währenddessen spricht Byles: „And I saw a new heaven and a new earth: for the first heaven and the first earth had passed away; and there was no more sea. And I John saw the holy city, new Jerusalem, coming down from God out of heaven, prepared as a bride adorned for her husband. And I heard a voice out of heaven saying, Behold, the tabernacle of God is with men, and he shall dwell with them, and they shall be his people, and God himself shall be with them, and be their God. And God shall wipe away all the tears from their eyes; and there shall be no more death, nor there shall be sorrow or crying, nor there shall be any more pain. The former world has passed away" (Offb 21,1-4). ${ }^{41}$ Einmal mehr fragt man sich angesichts solcher Worte: Wo ist

38 Anderen Überlieferungen zufolge spielten die Musiker „Autumn“; wieder andere Augenzeugen berichten, die Band hätte nicht bis zuletzt gespielt.

39 Siehe dazu auch: Riess, Richard: Der Untergang der Titanic. Pastoralpsychologische Aspekte des apokalyptischen Lebensgefühls in der heutigen Welt. In: Sommer, Wolfgang (Hg.): Zeitenwende - Zeitenende. Beiträge zur Apokalyptik und Eschatologie. Köln 1997, S. 149-175, hier S. 151 sowie Behr, Manfred: Philologisches Filmerleben. Literarische Dimensionen in Titanic. In: Gemeinschaftswerk der evangelischen Publizistik: Medien praktisch. Texte. Filmerleben. Zur emotionalen Dramaturgie von Titanic. Sonderheft Nr. 2 der Zeitschrift medien praktisch, September 1998, S. 52-64, hier S. 62.

40 Siehe dazu: Titanic Story Book: James Camerons illustriertes Drehbuch; ausführliches Interview mit Autor, Regisseur und Produzent James Cameron, kommentiert von Randall Frakes. Nürnberg 1999, S. XVII.

41 Der hier zitierte Wortlaut der Verse aus Offb 21,1-4 entspricht bis auf einige drehbuchbedingte Änderungen der King James Version (1611/1769): „And I saw a new heaven and a new earth: for the first heaven and the first earth were passed away; and there was no more sea. And I John saw the holy city, new Jerusalem, coming down from God out of heaven, prepared as a bride adorned for her husband. And I heard a great voice out of heaven saying, Behold, the tabernacle of God is with men, and he will dwell with them, and they shall be his people, and God himself shall be with them, and be their God. And God shall wipe away all tears from their eyes; 
Gott zu diesem Zeitpunkt, und wie kann er eine solche Katastrophe zulassen? So umkreist Camerons Film mehrmals die quälende Frage nach der Abwesenheit Gottes.

\section{Säkularisiertes Osterkerygma als Element der Glaubensbot- schaft "Titanic's"}

Die traumartige Schlußsequenz „Titanic's“ schließlich zeigt die gemäß der Interpretation Jacks als modernen Christus und Pater Byles verzweifelter Rezitation der Offenbarung des Johannes als Vorwegnahme des Zukünftigen folgerichtige Auflösung des Plots. In dieser vermengen sich der christliche Auferstehungsglaube und die Beschreibung der neuen, wunderbaren Welt Gottes nach der Apokalypse auf säkularisierte Weise: In den Bildern der Schlusssequenz, die eine durch Liebe gewirkte Auferstehung 42 zeigen, findet sich auch die Osterbotschaft im Glaubenssystem „Titanic's" wieder. ${ }^{43}$ Nachdem die 101-jährige Rose ihre Erlebnisse auf der Jungfernfahrt der „Titanic“ erzählt hat, geht sie zu Bett. Die anschließende Schlußsequenz des Filmes zeigt das Wrack der „Titanic“, das langsam zur neuen „Titanic“ wird (vgl. Offb 21,5a: „Er, der auf dem Thron saß, sprach: Seht, ich mache alles neu“). Eine Flügeltüre wird geöffnet (vgl. die Textpassage „once more you open the door" aus „My Heart Will Go On“). Sich öffnende Tore sind ein Ostersymbol. In der patristischen Exegese verweisen sich öffnende Tore auf den Einzug Christi in den Himmel (vgl. Ps 24,1-1044). Rose geht durch die geöffnete Tür. Auch die Lounge der Ersten Klasse, die Rose nun betritt, erstrahlt in neuem Glanz. Sie ist, dem himmlischen Jerusalem vergleichbar, nicht nur

and there shall be no more death, neither sorrow, nor crying, neither shall there be any more pain: for the former things are passed away" (Offb 21,1-4; King James Version [1611/1769]: Zit.n.: CD-Rom: Bibleworks ${ }^{\mathrm{TM}}$ for Windows 3.5 [Win 95/Win 3.1/NT] Databases. Published by Hermeneutika. Big Fork, Montana 1996).

42 Vgl. dazu den Text von „My Heart Will Go On“: „We'll stay forever this way. You are save in my heart and my heart will go on and on."

43 Zur Osterbotschaft in „Titanic" siehe auch: Vasel, Stephan: Kultkino und Religion. Christliches Erbe im Film am Beispiel „Titanic“. In: Materialdienst der Evangelischen Zentralstelle für Weltanschauungsfragen. 63. Jg. 12/2000, S. 411-428, hier S. $422 \mathrm{f}$ sowie ders.: Religiöse Dimensionen der Kulturindustrie. Christliche Motive in „Ben Hur" und „Titanic“. In: Loccumer Pelikan. Religionspädagogisches Magazin für Schule und Gemeinde. 1/1999, S. 26-29, hier S. 29.

44 Siehe dazu: Margoni-Kögler, Michael: Psalm 24 und Christi Himmelfahrt. Ein Beitrag zur patristischen Psalmenauslegung. Diplomarbeit Wien 1994. Zahlreiche Belegstellen aus der patristischen Literatur finden sich hier auf S. 52-115. 
wiederhergestellt, sondern erscheint sogar noch prachtvoller. ${ }^{45}$ Rose geht weiter zur großen Treppe, an der sie sich einst mit Jack zur Party im Unterdeck verabredet hatte. Der „wiederauferstandene“ Jack wartet erneut bei der Wanduhr auf Rose. Jack steht im Mittelpunkt des Raumes, „Titanic“-Passagiere aller gesellschaftlichen Klassen stehen rings um ihn (vgl. Offb 21,3b: „Er wird in ihrer Mitte wohnen, und sie werden sein Volk sein"). Die strenge Einteilung in Klassen wurde offensichtlich zugunsten eines sozial gerechteren Gesellschaftssystems aufgehoben, denn die Passagiere befinden sich nicht wie einst nach Klassen getrennt auf ihren jeweiligen Decks, sondern stehen jeder nach Belieben im Umkreis

Der Vergleich der Lounge mit dem himmlischen Jerusalem findet sich auch bei: Vasel, Stephan: Kultkino und Religion. Christliches Erbe im Film am Beispiel „Titanic“. In: Materialdienst der Evangelischen Zentralstelle für Weltanschauungsfragen. 63. Jg. 12/2000, S. 411-428, hier S. 422 sowie bei dems.: Religiöse Dimensionen der Kulturindustrie. Christliche Motive in „Ben Hur" und „Titanic“. In: Loccumer Pelikan. Religionspädagogisches Magazin für Schule und Gemeinde. 1/1999, S. 26-29, hier S. 29. Manfred Behr beschreibt diesen Vergleich nicht, findet stattdessen jedoch interessanterweise Parallelen einer früheren, ebenfalls in der Lounge der Ersten Klasse stattfindenden Einstellung zum himmlischen Jerusalem. Es ist dies die ungewöhnliche, nach oben gefilmte Unterwasseraufnahme einer ertrunkenen Frau in einem weißen Kleid, „die vor dem Hintergrund des Kronleuchters in der Lounge der Ersten Klasse gespenstisch im Wasser kreist“" (Cameron, James. In: Titanic Story Book: James Camerons illustriertes Drehbuch; ausführliches Interview mit Autor, Regisseur und Produzent James Cameron, kommentiert von Randall Frakes. Nürnberg 1999, S. 134). In ihrem zerfließenden Gewand, so Behr, gemahnt diese Frau „tatsächlich an die geschmückte Braut, die aber nun tot ist und mit weit ausgebreiteten Armen vor den Sternen des Himmelgewölbes treibt" (Behr, Manfred: Philologisches Filmerleben. Literarische Dimensionen in Titanic. In: Gemeinschaftswerk der evangelischen Publizistik: Medien praktisch. Texte. Filmerleben. Zur emotionalen Dramaturgie von Titanic. Sonderheft Nr. 2 der Zeitschrift medien praktisch, September 1998, S. 52-64, hier S. 62). Der Kronleuchter als Himmlisches Jerusalem, merkt Behr weiter an, ist seit der Gotik Teil christlicher Ikonografie. Der Verweis der Lounge auf das himmlische Jerusalem verdichtet sich, denkt man an das Filmende, in dem Rose und Jack - so die Interpretation der Schlussszene unter Beachtung ihrer dichten Symbolisierungen - im Tod vereinigt sind, richtet sich doch auch hier die Kamera auf den Kronleuchter der Halle, bevor das Bild in eine Weißblende übergeht. James Cameron setzte die Einstellung der im Wasser treibenden Frau direkt hinter die Szene der Bibelrezitation von Pater Byles aus der Offenbarung des Johannes, da er fand, „daß sie dort besonders eindringlich wirke“ (Titanic Story Book: James Camerons illustriertes Drehbuch; ausführliches Interview mit Autor, Regisseur und Produzent James Cameron, kommentiert von Randall Frakes. Nürnberg 1999, hier S. 134). Der Vers aus der Offenbarung des Johannes, den Pater Byles spricht, lautet: „Ich sah die heilige Stadt, das neue Jerusalem, von Gott her aus dem Himmel herabkommen; sie war bereit wie eine Braut, die sich für ihren Mann geschmückt hat“ (Offb 21,2). 
von Jack und der zu ihm eilenden Rose und Jack trägt wieder seine eigene und nicht die einst für das Auftreten in der Ersten Klasse ausgeliehene Kleidung.

So bewahrheitet sich tatsächlich, was uns Pater Byles auf dem Höhepunkt der Schiffskatastrophe wissen läßt. Im einzelnen heißt das:

- Byles sprach: „And I saw a new heaven and a new earth: for the first heaven and the first earth had passed away; and there was no more sea." Der erste Himmel und die erste Erde sind mit der "Titanic" in den Fluten versunken. Es muss daher ein neuer Himmel bzw. eine neue Erde sein, wo Jack und Rose in der Schlusssequenz des Filmes einander wiedersehen. Dass es dort kein Meer mehr gibt, impliziert die angesichts der „Titanic“-Katastrophe wahrlich beruhigende Aussicht, dass sie nicht noch einmal Schiffbruch erleiden können und von nun an vereint sein werden.

- Byles zitierte weiter: „And I John saw the holy city, new Jerusalem, coming down from God out of heaven, prepared as a bride adorned for her husband." Wie oben beschrieben steht in der Schlusssequenz „Titanic's“ die Lounge der ersten Klasse symbolisch für das neue Jerusalem.

- Byles fuhr fort: „And I heard a voice out of heaven saying, Behold, the tabernacle of God is with men, and he shall dwell with them, and they shall be his people, and God himself shall be with them, and be their God." Die Jesus-Figur Jack steht im Mittelpunkt der Lounge umgeben von "seinem“ jubelnden Volk, den beim Untergang der "Titanic" verstorbenen Passagieren.

- Byles weiter: „And God shall wipe away all the tears from their eyes; and there shall be no more death, nor there shall be sorrow or crying, nor there shall be any more pain. The former world has passed away." Aller Kummer und Schmerz, den die "Titanic"-Passagiere erleiden mussten, sind vorbei. Die Toten sind auferstanden. Sie befinden sich in einer neuen Seinsebene, einer "neuen Welt".

Der Tod wird im Glauben fast aller Völker als Übergang oder als Reise in eine andere Welt empfunden. ${ }^{46}$ Auch als eine Art Zwischenreich und als Tor zum größeren Leben wird der Tod gesehen. Dieser Glaubensansicht folgt James Cameron, indem er durch das Filmende das „größere Leben" andeutet, das Jack und Rose nach dem Tod erwartet - "größer"

46 Lurker, Manfred: Die Botschaft der Symbole. In Mythen, Kulturen und Religionen. München 1990, S. 243 sowie Fromm, Erich: Märchen, Mythen, Träume. Eine Einführung in das Verständnis einer vergessenen Sprache. Reinbek bei Hamburg 1998, S. 54. 
insofern, als die beiden ihre Liebe nun offen leben dürfen, was durch den Beifall der anderen Passagiere verdeutlicht wird.

„Der Mensch“, so Hartmut Heuermann, „wird erlöst, indem er den Tod als mythische Wandlung zu neuem Leben begreift." 47 Diesem Gedankengang folgend, bietet „Titanic“ einen Weg der Erlösung: Zuerst fängt nach Jacks Tod Roses neues Leben an, und schließlich beginnt, so Camerons Andeutung, nach Roses Tod Jacks und Roses gemeinsames neues Leben im „neuen Jerusalem“, umgeben von den einst beim Untergang der „Titanic" Verstorbenen. Erlösung bzw. Läuterung findet auch der Schatzsucher Brock Lovett, der durch Roses Geschichte tief bewegt die menschlichen Tragödien angesichts des Untergangs der „Titanic“ nachvollziehen kann und reumütig die Plünderungen des Wracks beendet. Der Reeder Bruce Ismay hingegen, der die „Titanic“ konstruierte und Kapitän Smith in Camerons Film zu einer Geschwindigkeitserhöhung überredete, ist eine moderne Version des Judas, denn Ismay kann die Kreuzigung derer, an denen er sich schuldig gemacht hat, nicht mitansehen, steigt in ein Rettungsboot, blickt weg und hofft, nicht aufzufallen.

\section{Resümee}

James Camerons "Titanic" zeigt menschliche Schicksale, Geschichten von Schuld, innerer Befreiung, Sinnfindung sowie einen breiten Fächer unterschiedlichen Sterbeverhaltens - all dies eingebettet in ein Glaubenssystem, in dem die alles, selbst den Tod, überwindende Liebe als bedeutendster Sinnfaktor gilt und die menschliche Hybris angeprangert wird. Die Position Gottes wird hier weitgehend von der allmächtigen, die Hybris bestrafenden Natur eingenommen. Dabei inszeniert Cameron den götzenhaften Glauben an Technik und Fortschritt als spezifisch männliche Hybris und betont das Ausgeliefertsein des Menschen an geheimnisvolle Mächte. Auch die Vorstellung vom Untergang des Bösen und vom Sieg des Guten und damit einer letzten Gerechtigkeit sowie das Versprechen auf eine bessere Welt, in der Werte wie Liebe und Geborgenheit herrschen, bekommen als zuversichtliche Hoffnung Platz im Glaubenssystem „Titanic's“. Zu dieser sinnstiftenden und alltagstranszendierenden Funktion kommt noch der Orientierungs- und Leitbildcharakter von James Camerons "Titanic“. In Zeiten, in denen ein Glaubens-Motto der westlichen Welt „Seeing is believing“ lautet, d.h. nicht

47 Heuermann, Hartmut: Medien und Mythen. Die Bedeutung regressiver Tendenzen in der westlichen Medienkultur. München 1994, S. 122. 
Zeigbares zusehends als nicht glaubbar erlebt wird, trifft die Glaubensbotschaft „Titanic's“ genau den Kern der religiösen Lage. Überhaupt kommt medialen Orientierungsangeboten gegenwärtig „ëine wachsende Bedeutung zu. Sie werden $z u$ Sinnressourcen, weil sie in ihren Erzählungen einerseits gesellschaftlich virulente Alltagsmythen reartikulieren und andererseits als kollektives Event-Erlebnis eine wachsende Verbindlichkeit solcher Alltagsmythen suggerieren. “48

Zudem trifft die Botschaft von einer Liebe, die stärker ist als der Tod zugleich ein klassisches Thema vieler Religionen - in einer Welt, die Liebe und Emotionalität häufig Maximen wie wirtschaftlicher Effizienz und Gewinnmaximierung unterwirft, genau in das emotionale Vakuum der westlichen Gesellschaft. Weiter wird zur Entlastung der Zuschauer in Camerons Film nicht die Theodizeefrage, sondern die Anthropodizeefrage behandelt: Es geht nicht in erster Linie darum zu fragen, wie Gott eine derartige Katastrophe zulassen konnte, sondern darum, wie die Menschen sich angesichts einer solchen Katastrophe zu verhalten haben. So bleibt die Klage gegen Gott aus. Auch die Liebe und das Vertrauen, die Jack und Rose füreinander empfinden, überwölben die Liebe und das Vertrauen zu Gott. Erlösung bedeutet daher im Kontext dieses Filmes kein ewiges Bei-Gott-Sein, sondern das ewige Zusammensein mit dem geliebten Menschen.

Der christliche Heilsoptimismus wird im Glaubenssystem „Titanic's“ säkularisiert und gedrosselt und dadurch einem breiten Publikum zugänglich gemacht. So weist das Glaubenssystem von James Camerons „Titanic“ zwar deutliche Parallelen zu christlichem Glaubensgut und biblischen Szenen auf, spielt sich dabei aber stets auf rein menschlicher Ebene ab. Aus diesem Grund kann jeder, ob in religiösem Sinn gläubig oder nicht, in dem Film eine Glaubensbotschaft finden, die ihn anspricht.

Dass dies zumeist auf einer unbewußten Ebene geschieht, tut der Tatsache keinen Abbruch, dass die religiösen Elemente in „Titanic“ mit Sicherheit maßgeblich zum Erfolg dieses Filmes beitrugen. Im Gegenteil: Popularfilme wie „Titanic“ plazieren ihre Sinnparadigmen gezielt auf der Subtextebene, denn schließlich ist es weder primäres Ziel der Filme, Seelsorge zu leisten, noch ist Sinndeutung ein bewusstes Motiv für den Kinobesuch. Aufgrund dieser Kombination von nicht augenscheinlichem

48 Hepp, Andreas/Vogelsang, Waldemar: ,Ich hab' einfach nur geheult'. Zur emotionalen Aneignung des Medien-Events Titanic aus Rezipientenperspektive. In: Gemein schaftswerk der evangelischen Publizistik: Medien praktisch. Texte. Filmerleben. Zur emotionalen Dramaturgie von Titanic. Sonderheft Nr. 2 der Zeitschrift medien praktisch, September 1998, S. 30-40, hier S. 39. 
Anbieten und nicht augenscheinlichem Suchen nach Antworten auf letzte Fragen wurde die auf latenter Ebene gebotene Undercover-Religion „Titanic's“ weltweit von so vielen Menschen - meist ebenfalls auf latenter Ebene - angenommen, dass der Film zum meistgesehenen Kinospielfilm aller Zeiten avancierte. 